\title{
The Pain Course: exploring the feasibility of an internet-delivered pain management programme for adults with spinal cord injury
}

\author{
B. F. Dear ${ }^{1} \cdot$ K. Nicholson Perry $\mathbb{1}^{2} \cdot$ P. Siddall $\mathbb{C}^{3,4} \cdot$ J. W. Middleton ${ }^{4,5} \cdot$ J. Johnson ${ }^{6} \cdot$ L. Katte ${ }^{7} \cdot$ F. Monypenny ${ }^{5} \cdot$ \\ E. Karin $\mathbb{D}^{1} \cdot$ M. Gandy ${ }^{1} \cdot$ N. Titov ${ }^{1}$
}

Received: 13 February 2018 / Revised: 17 April 2018 / Accepted: 17 April 2018 / Published online: 31 May 2018

(c) International Spinal Cord Society 2018

\begin{abstract}
Study design Single-group feasibility clinical trial.

Objectives This study examined the feasibility and outcomes of a modified version of a validated internet-delivered pain management programme, the Pain Course, for adults with SCI.

Setting Nationwide in Australia.

Methods Sixty-eight adults participated in the programme, which comprises five online lessons and homework tasks that are systematically released over 8 weeks. Participants were supported through the course with weekly contact from a clinical psychologist.

Results Eighty-five percent of participants provided data at post-treatment and $76 \%$ of participants completed all five lessons of the course. High levels of satisfaction were observed and relatively little clinician time $(\mathrm{M}=93.16 \mathrm{~min} ; \mathrm{SD}=52.76 \mathrm{~min})$ was required per participant to provide the course. Preliminary evidence of clinical improvements in pain-related disability ( $d s \geq 0.53$; avg. improvement $\geq 20 \%$; Mdiff $\geq 7.77$ ), depression ( $d s \geq 0.44$.; avg. improvement $\geq 24 \%$; Mdiff $\geq 2.44$ ), anxiety ( $d s \geq 0.41$; avg. improvement $\geq 26 \%$; Mdiff $\geq 1.8$ ) and average pain intensity ( $d s \geq 0.46$; avg. improvement $\geq 13 \%$; Mdiff $\geq 0.71$ ) were observed at post-treatment, which were maintained or further improved to 3-month follow-up. These improvements were reflected in overall improvements in self-reported satisfaction with life $(d s \geq 0.31$; avg. improvement $\geq$ $25 \%$; Mdiff $\geq 2.16$ )
\end{abstract}

Conclusion These findings highlight the potential of carefully developed internet-delivered interventions as an approach for overcoming barriers and increasing access to psychosocial care for adults with SCI.

Sponsorship iCare Lifetime Care and Support Authority and the Australian National Health and Medical Research Council.

B. F. Dear

blake.dear@mq.edu.au

1 eCentreClinic, Department of Psychology, Macquarie University, Sydney, NSW, Australia

2 Discipline of Psychological Sciences, Australia College of Applied Psychology, Sydney, NSW 2000, Australia

3 Department of Pain Management,Greenwich Hospital, HammondCare, Sydney, NSW, Australia

4 Sydney Medical School-Northern, University of Sydney, Sydney, NSW 2006, Australia

5 State Spinal Cord Injury Service, NSW Agency for Clinical Innovation, Chatswood, Sydney, NSW, Australia

6 Pain Management Network, NSW Agency for Clinical Innovation, Chatswood, Sydney, NSW, Australia

7 NSW Spinal Outreach Service, Royal Rehab Centre, Ryde, Sydney, NSW, Australia

\section{Introduction}

Spinal cord injury (SCI) can occur as a result of traumatic injury (e.g., motor vehicle accident, workplace and sporting accidents, falls and assault) or due to non-traumatic disease processes (e.g., tumour, infection, and infarction). SCI results in impairments of sensorimotor and autonomic functions below the level of the injury. Secondary health complications are common as a result including altered bladder and bowel functioning, difficulties with sexual dysfunction, increased risk of urinary tract infections and pressure injuries [1-4]. Chronic pain is one common consequence of SCI, with up to $80 \%$ experiencing either persistent neuropathic and/or musculoskeletal pain [5]. High rates of depression and anxiety are also common $[6,7]$, especially in the context of persistent pain and other secondary complications $[8,9]$. 
There is encouraging evidence for psychological interventions for adults with SCI, particularly those based on the principles of cognitive behaviour therapy for mental health conditions [10, 11] and chronic pain [12-14]. However, people with SCI face numerous barriers to accessing such psychologically based interventions, including cost, mobility restrictions and limited availability of clinicians with the necessary expertise, especially outside of major cities [1517]. This has led to interest in the remote delivery of psychological treatment via telephone [17] and the internet [18-20] as a way to increase access to care.

A significant body of research has emerged over the last decade supporting the internet delivery of psychological treatments for anxiety and depression [21], as well as chronic pain [22]. These internet-delivered interventions are based on the same principles as face-to-face treatments, providing the same therapeutic information and teaching the same self-management skills. However, these interventions use carefully developed online modules, which people can work through themselves without leaving their home, to provide this information and teach these skills. Internetdelivered interventions are often provided with regular clinician contact via telephone and secure email, where the clinician provides encouragement, support, answers questions and helps to tailor the intervention for the patient $[23,24]$. However, they can also be provided with very little or no clinician support [23].

Two small feasibility studies [18, 19] and one randomised controlled trial (RCT) [20] have examined the potential of internet-delivered interventions for depression among adults with SCI. The feasibility studies (combined $n$ $=17$ ) found evidence of acceptability and some evidence of improvements in symptoms of depression and quality of life $[18,19]$. The RCT $(n=48)$ found small improvements in depression, anxiety, stress and satisfaction with life in the treatment group, and improvements in depression, but no other outcomes, in the control group [20]. However, adherence has been noted as an issue, with most participants completing less than half of the intervention.

To date, no studies have examined the potential of emerging internet-delivered interventions for chronic pain among adults with SCI. Some clinical trials of wellestablished internet-delivered interventions for chronic pain, such as the Pain Course, have included small numbers of adults with SCI and found improvements in levels of disability, depression, anxiety and pain [23-25]. However, these studies have focused on people with a broad range of different pain-related conditions and have not reported results specifically for adults with SCI. Thus, it is unclear whether such interventions are acceptable or effective for adults with SCI. Targeted clinical trials are needed to address this gap.
The present study sought to evaluate the feasibility and preliminary outcomes of a modified version of a validated internet-delivered intervention for chronic pain, the Pain Course, for adults with SCI. Consistent with previous trials, it was hypothesised that: (1) the programme would be highly acceptable and that high levels of engagement would be observed; (2) the programme would require relatively little clinician time to administer; and (3) preliminary evidence of improvements in levels of disability, anxiety, depression and pain would be observed and maintained to 3-month follow-up.

\section{Methods}

\section{Participants}

Potential participants read about the trial and applied to participate via the eCentreClinic website (www. ecentreclinic.org). The eCentreClinic is a specialist Australian research unit that offers Australian adults the opportunity to participate in clinical trials and receive access to free self-management programmes for a range of conditions, including chronic pain. The eCentreClinic website can be located via online searches and is promoted by various health professionals and health-related websites within Australia. The present trial was also promoted via the New South Wales (NSW) Government's Agency for Clinical Innovation Pain Management Network and the NSW State Spinal Cord Injury Service.

Those expressing interest completed an online screening questionnaire to ensure they met the inclusion and exclusion criteria, which were then confirmed during a subsequent telephone assessment. Inclusion criteria were: (a) diagnosed with a SCI; (b) experiencing chronic pain for $>6$ months; (c) had a consultation with a GP or specialist in the previous 6 months who is managing their care; (d) 18 years or older; and (e) living in Australia at the time of the trial. Exclusion criteria were: (a) very severe symptoms of depression (total Patient health questionnaire 9-item (PHQ-9) score $\geq 22$ ) or suicidal ideation (a score of 3 on question 9 of the PHQ-9); (b) recent history (within the last 12 months) of attempted suicide or self-harm.

A total of 156 people started an online application to participate in the trial between May 2015 and June 2017. Of these, 70 met all of the inclusion and exclusion criteria upon telephone assessment, and were allocated to the study. Sixty-eight participants started the intervention and were included in the evaluation. Details of participant flow into the study are provided in Fig. 1. 


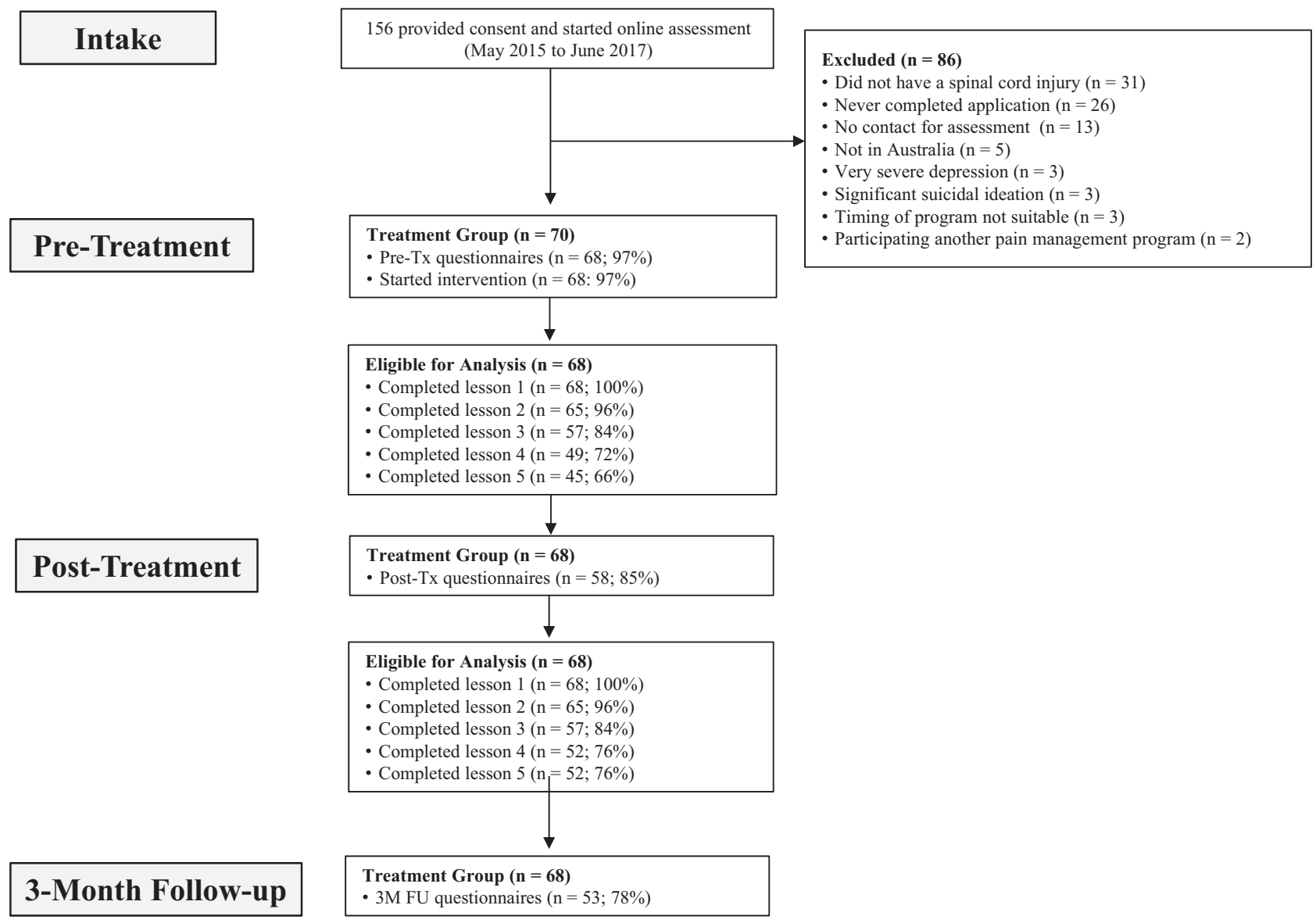

Fig. 1 Participant flow from application to 3-month follow-up

\section{Study design and measures}

A prospective longitudinal single-group open-trial design was employed. The acceptability and satisfaction questions were administered at post-treatment only. The primary and secondary outcomes were assessed at initial application, pre-treatment, mid-treatment, post-treatment (i.e., 9 weeks after pre-treatment) and 3-month follow-up. To reduce burden, tertiary outcomes were not assessed at initial application or mid-treatment. The trial was approved by the Macquarie University Human Research Ethics Committee and was registered on the Australian and New Zealand Clinical Trials Registry: ACTRN12614000767606.

\section{Primary measures}

Pain disability index (PDI) The PDI contains seven items designed to measure the impact of pain on: (a) family and home responsibilities; (b) recreation; (c) social activity; (d) occupation; (e) sexual behaviour; (f) self-care; and (g) life support activities [26]. Scores range from 0 to 70 and higher scores indicate greater impact and disability due to pain. In the present sample, Cronbach's $\alpha=0.86$.
Patient health questionnaire 9-item The PHQ-9 contains nine items, which measure the symptoms and severity of depression [27]. Scores range from 0 to 27 and higher scores indicate greater depression symptom severity. A total score of $\geq 10$ is indicative of a DSM-IV diagnosis of depression. In the present sample, Cronbach's $\alpha=0.82$.

Generalised anxiety disorder scale 7-item (GAD-7) The GAD-7 contains seven items designed to measure symptoms and severity of anxiety [28]. Scores range from 0 to 21 and higher scores indicating greater severity of anxiety symptoms. A total score of $\geq 10$ is indicative of a DSM-IV diagnosis of an anxiety disorder. In the present sample, Cronbach's $\alpha=0.89$.

\section{Secondary measure}

Wisconsin brief pain questionnaire (WBPQ) The four items of the WBPQ concerning participant's current, average, least and worst pain intensities over the last month were employed [29]. Each item comprises an 11-point visual analogue scale (VAS) scale, with scores ranging from 0 to 
10 and higher scores indicate worse pain. Only participants' average pain intensity levels are reported here.

\section{Tertiary measures}

Pain self-efficacy questionnaire (PSEQ) The PSEQ contains 10 statements regarding a patient's beliefs about his or her ability to undertake a number of daily tasks with pain [30]. Scores range from 0 to 60 and higher scores indicate greater pain-related self-efficacy. In the present sample, Cronbach's $\alpha=0.90$.

Pain catastrophising scale (PCS) The PCS contains 13 items designed to measure the tendency to magnify the threat value of ongoing pain [31]. Scores range from 0 to 52 and higher scores indicating a greater tendency to magnify the threat and significance of pain. In the present sample, Cronbach's $\alpha=0.93$.

The satisfaction with life scale (SWLS) The SWLS is a fiveitem measure of perceived satisfaction with one's life [32]. Scores range from 5 to 35 and higher scores indicating greater life satisfaction. In the present sample, Cronbach's $\alpha=0.83$.

\section{Acceptability and satisfaction}

Consistent with previous research [7-10, 15], treatment satisfaction and acceptability was assessed at post-treatment via three questions: (1) 'Overall, how satisfied were you with the course?', (2) 'Would you feel confident in recommending this course?', and (3) 'Was it worth your time doing the course?' Participants responded to the first question using a five-point Likert scale, which ranged from 'very satisfied' to 'very dissatisfied', and the second two questions with a 'yes' or 'no' response.

\section{Treatment programme}

The Pain Course is an efficacious internet-delivered pain management programme based on principles of transdiagnostic cognitive behaviour therapy and is described in detail elsewhere [23-25, 33, 34]. The course is based on established face-to-face pain management programmes, providing the same therapeutic information to support participants to learn cognitive and behaviour self-management skills. Thus, it is designed to be suitable for adults with a broad range of different pain conditions and types of pain. It consists of five core online lessons and five downloadable lesson summaries, which are released over 8 weeks. Each lesson is presented in the form of a slide show, which takes approximately $10-20 \mathrm{~min}$ to read, and is designed to be easily read by someone without high school reading proficiency. The lesson materials are presented in a didactic format and include realistic examples of skills practice and symptom management, which are strategically integrated throughout the lessons to aid learning. Additional resources are provided to introduce further topics and skills that are relevant for many participants. Several detailed case stories and numerous real-world examples are also provided to demonstrate how to apply the information and skills covered in the course. There was no time-limit for accessing or reading the course. Participants were encouraged to spend at least $4 \mathrm{~h}$ a week working on the course and practicing the skills taught. An overview of the structure, content and skills taught within the Pain Course is provided in Table 1.

The Pain Course was modified slightly for the current trial to include case stories and examples of skill use from adults with SCI. Basic information about autonomic dysreflexia, and the prevalence and characteristics of chronic pain following SCI was also added. The course was accessed via secure online login and all participants were provided their own personal account and password. Participants were sent regular automated emails throughout the course; (i) advising them when new content was available, (ii) reminding them when there was content they had not accessed, (iii) congratulating them when they completed a lesson, and (iv) normalising difficulties and encouraging skills practice.

\section{Clinical contact}

One senior clinical psychologist (BFD) with postgraduate qualifications and $>10$ years' clinical experience in chronic pain management provided all clinical contact. Consistent with previous research [23-25, 33, 34], the clinician focused contact on: (i) answering participants' questions; (ii) summarising content; (iii) encouraging skills practice and reinforcing progress; (iv) enquiring about participants' experiences with the course and use of the skills; and (v) normalising challenges in the learning and use of the core skills. The clinician attempted to contact participants each week of the course, via telephone or a secure email system, and tried to limit the time spent in contact with participants to approximately $10-15 \mathrm{~min}$ per week.

\section{Analytic plan}

All analyses were conducted using SPSS version 24 . Generalised estimation equation (GEE) modelling was employed to examine changes over time from baseline to 3-month follow-up [35]. Initial application data were employed as baseline for the primary and secondary outcomes, and pre-treatment data were used as baseline for the tertiary outcomes. An unstructured working correlation structure was applied to account for within subjects' 


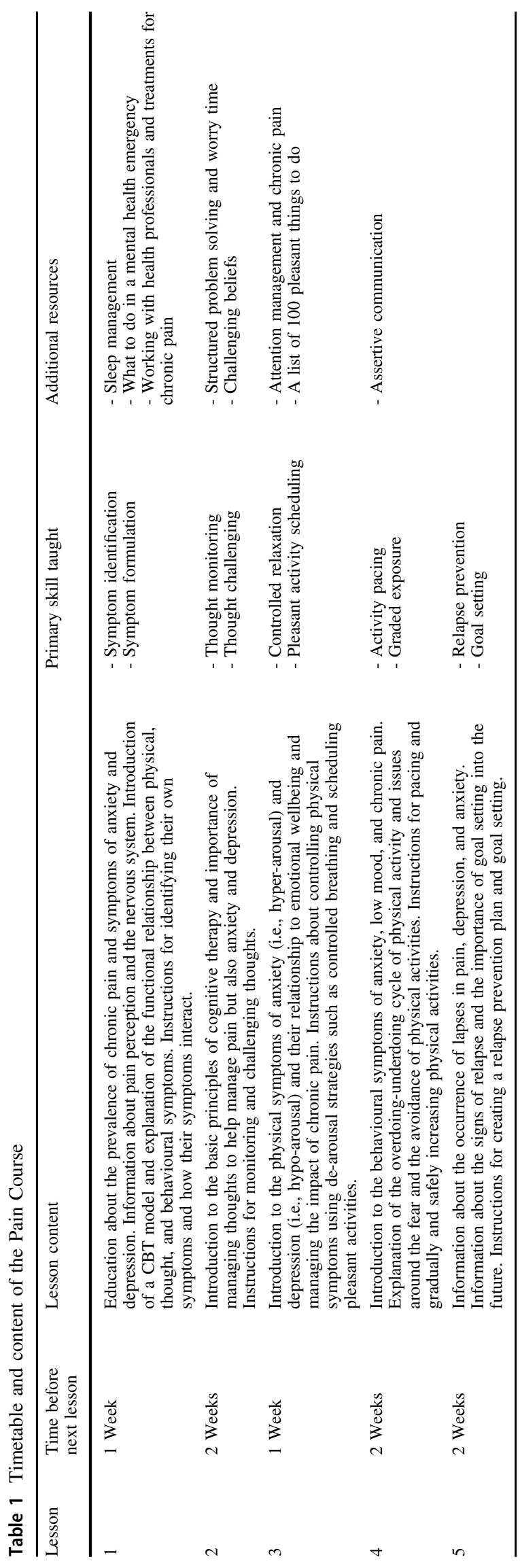

variances over time. A gamma distribution with a log link response scale were specified to address positive skewness and proportionally changing scores in the dependent variable. Pairwise comparisons were used to examine the statistical significance of changes in the outcomes between the time points. Importantly, adjusted longitudinal GEE models were used to impute the data of missing cases under intention-to-treat principles. Consistent with expert recommendations [36], these adjusted GEE models accounted for participants' symptom levels at baseline, as well as the number of treatment modules completed. Consistent with the earlier reports [23-25, 33, 34], clinical significance was assessed in two ways. First, average percentage change over time was calculated, using the $\exp (\beta)$ change factors derived from the GEE models. Importantly, to accurately reflect percentage change, a constant of 6 was subtracted from SWLS scores when calculating percentage change scores to result in a minimum score of 0 . Second, Cohen's $d$ effect sizes were calculated, based on the estimated marginal means. Mean difference (Mdiff) scores between pretreatment and post-treatment and pre-treatment and 3month follow-up are also reported.

\section{Results}

\section{Baseline data and attrition}

The demographic and clinical characteristics of the sample are shown in Table 2 and Table 3. Specific details of participant flow, treatment attrition, lesson completion, and questionnaire response are shown in Fig. 1. Data were collected from $85 \%$ and $78 \%$ of participants at posttreatment and 3-month follow-up, respectively.

\section{Treatment engagement and acceptability}

In all, $66 \%$ and $76 \%$ of participants completed all five lessons by post-treatment and 3-month follow-up, respectively. Of the participants completing the treatment satisfaction questions, $89 \%$ (50/56) reported being satisfied or very satisfied with the course and no one reported being dissatisfied or very dissatisfied. Similarly, $100 \%$ (56/56) reported they would recommend the course to others and 96\% (54/56) reported the course was worth their time.

\section{Time spent and summary of contacts}

The mean total clinician contact time per participant was $93.16 \mathrm{~min}(\mathrm{SD}=52.76)$, which consisted of answering and making calls $(\mathrm{M}$ time $=65.72 ; \mathrm{SD}$ time $=54.00)$ and sending or reading secure emails $(\mathrm{M}$ time $=27.44, \mathrm{SD}$ time $=13.12$ ). 
Table 2 Demographic characteristics of the sample

\begin{tabular}{|c|c|c|}
\hline Variables & $n$ & $\%$ \\
\hline \multicolumn{3}{|l|}{ Gender } \\
\hline Male & 34 & $50 \%$ \\
\hline Female & 34 & $50 \%$ \\
\hline \multicolumn{3}{|l|}{ Age } \\
\hline Mean & $48(13)$ & \\
\hline Range & $21-85$ & \\
\hline \multicolumn{3}{|l|}{ Marital status } \\
\hline Single & 20 & $29 \%$ \\
\hline Married/de facto & 37 & $54 \%$ \\
\hline Separated/divorced/widowed & 11 & $16 \%$ \\
\hline \multicolumn{3}{|l|}{ Education } \\
\hline High school or less & 17 & $25 \%$ \\
\hline Certificate/diploma/other ${ }^{\mathrm{a}}$ & 27 & $39 \%$ \\
\hline University & 24 & $35 \%$ \\
\hline \multicolumn{3}{|l|}{ Employment status ${ }^{\mathrm{b}}$} \\
\hline Employed full-time & 9 & $13 \%$ \\
\hline Employed part-time or casual & 14 & $20 \%$ \\
\hline Unemployed & 11 & $16 \%$ \\
\hline Registered disability & 24 & $35 \%$ \\
\hline Retired & 13 & $19 \%$ \\
\hline
\end{tabular}

Standard deviations are shown in parentheses. All data were selfreported

${ }^{a}$ These are qualifications obtained outside of high school in Australia but are less academically demanding than a university degree

${ }^{\mathrm{b}}$ Categories are not mutually exclusive; participants were able to select more than one category to describe their employment status

\section{Primary outcomes}

The means, standard deviations, percentage reductions, and Cohen's $d$ effect sizes for the primary, secondary and tertiary outcome variables for the overall sample are shown in Table 4. The GEE analyses revealed a significant overall time effect for pain-related disability (Wald's $\chi^{2}=55.50$, $p<0.001$ ), depression (Wald's $\chi^{2}=70.84, p<0.001$ ), and anxiety (Wald's $\chi^{2}=36.17, p<0.001$ ). Pairwise comparisons revealed significant improvements in disability, depression, and anxiety levels from baseline to posttreatment $(p<0.001)$, which further improved from posttreatment to 3-month follow-up $(p<0.015)$. These improvements corresponded to clinically meaningful improvements as shown in Table 4.

\section{Secondary outcomes}

The GEE analyses also revealed a significant overall time effect for average pain intensity (Wald's $\chi^{2}=30.72, p<$ 0.001). Pairwise comparisons indicated significant improvements in average pain levels from baseline to post-
Table 3 Injury and pain characteristics of the sample

\begin{tabular}{|c|c|c|}
\hline Variables & $n$ & $\%$ \\
\hline \multicolumn{3}{|l|}{ Time since SCI (years) } \\
\hline Mean & $8(10)$ & \\
\hline Range & 0.33 to 48 & \\
\hline \multicolumn{3}{|l|}{ SCI type } \\
\hline Complete paraplegia & 10 & $15 \%$ \\
\hline Incomplete paraplegia & 33 & $49 \%$ \\
\hline Complete tetraplegia & 5 & $7 \%$ \\
\hline Incomplete tetraplegia & 11 & $16 \%$ \\
\hline Unsure (type not formally diagnosed) & 9 & $13 \%$ \\
\hline \multicolumn{3}{|l|}{ SCI cause } \\
\hline Accident (at home, sporting) & 15 & $22 \%$ \\
\hline Workplace accident & 14 & $20 \%$ \\
\hline Motor vehicle accident & 18 & $26 \%$ \\
\hline Surgical or other medical treatment & 5 & $7 \%$ \\
\hline Disease or illness & 12 & $17 \%$ \\
\hline Deterioration of spine & 4 & $5 \%$ \\
\hline \multicolumn{3}{|l|}{ Pain duration (years) } \\
\hline Mean & $8(7)$ & \\
\hline Range & $0.5-32$ & \\
\hline Average number pain sites & $3.96(1.56)$ & \\
\hline Pain always present & 59 & $87 \%$ \\
\hline \multicolumn{3}{|l|}{ Pain location ${ }^{\mathrm{a}}$} \\
\hline Head/face/mouth & 8 & $12 \%$ \\
\hline Throat/neck/shoulders & 33 & $49 \%$ \\
\hline Arms/forearms/hands & 34 & $50 \%$ \\
\hline Chest/abdomen/pelvis & 34 & $50 \%$ \\
\hline Upper back/lower back & 53 & $78 \%$ \\
\hline Buttocks/hips/anus & 38 & $56 \%$ \\
\hline Upper leg/lower leg/feet & 56 & $82 \%$ \\
\hline \multicolumn{3}{|l|}{ Prescription medications reported ${ }^{a, b}$} \\
\hline Strong opioid analgesics & 32 & $47 \%$ \\
\hline Weak opioid analgesics & 16 & $24 \%$ \\
\hline Anticonvulsants & 41 & $60 \%$ \\
\hline Antispasmodics & 14 & $21 \%$ \\
\hline Benzodiazepines & 5 & $7 \%$ \\
\hline Anxiolytics and antidepressants & 29 & $43 \%$ \\
\hline Other pain or psychotropic medication & 14 & $21 \%$ \\
\hline
\end{tabular}

Standard deviations are shown in parentheses. All data were selfreported. All numbers rounded to nearest whole number

${ }^{\text {a}}$ Participants could endorse multiple responses

${ }^{\mathrm{b}}$ Only prescription medications for pain, a pain-related condition, anxiety, or depression are reported. Strong opioids: buprenorphine, fentanyl, hydromorphone, methadone, morphine, oxycodone. Weak opioids: codeine, tramadol, tapentadol. Anxiolytics and antidepressants: selective serotonin reuptake inhibitors, norepinephrine and serotonin-norepinephrine reuptake inhibitors, tricyclics and tetracyclics. Other psychotropic or pain medications: including corticosteroids, NSAIDS, DMARS, serotonin agonists, dopamine agonists, antipsychotics, and psychostimulants 


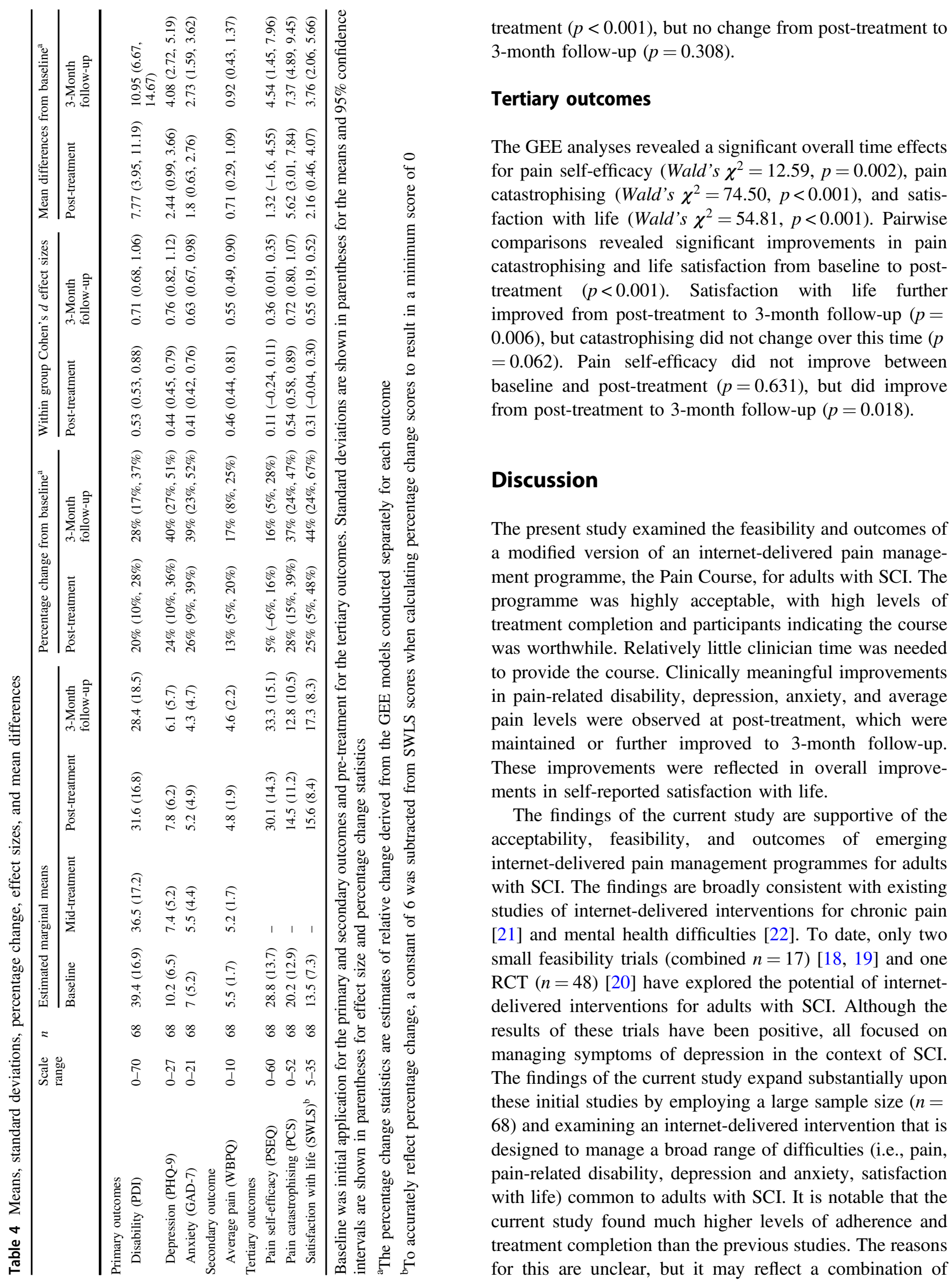


factors, including the brief nature of the current intervention, the fact the intervention dealt with a broad range of difficulties, and had been developed over numerous previous clinical trials. The findings of the current study highlight the promise of emerging internet-delivered interventions for adults with SCI and provide the necessary empirical support for further large scale research exploring their use.

There were several other notable findings from the current study. First, consistent with other studies [23-25, 33, 34], the current findings indicate these interventions can be administered to adults with SCI in a way that requires relatively little clinician time compared to traditional face-toface interventions. This highlights the potential costeffectiveness of these programmes, especially if the indirect costs and burden of having adults with SCI attend faceto-face programmes are considered [26]. Second, improvements were also observed on the important tertiary outcomes of pain self-efficacy, pain catastrophising, and satisfaction with life. However, some caution is needed around pain selfefficacy as it was found to improve in the period after the course, rather than during treatment as in previous studies not focussed on SCI [23, 24, 33]. Nevertheless, the improvements in pain catastrophising and satisfaction with life were significant and consistent with previous studies. Finally, the current study found evidence of acceptability, feasibility, and effectiveness despite recruiting a heterogeneous sample of participants with different types of SCI (e.g., complete and incomplete injuries) from a broad range of causes (e.g., traumatic and non-traumatic), and included participants with and without clinical significant levels of anxiety and depression.

The findings of this trial should be considered in the context of a number of important limitations. First, the absence of a control group means it is not possible to determine effects of the treatment beyond that of spontaneous remission. However, spontaneous remission would seem an unlikely explanation for the findings given the average duration of participants' pain and the persistent nature of chronic pain in the context of SCI. Second, although relatively large compared with existing studies, the sample size of the current study is still too small to explore the impact of potential clinical variables on clinical response, or to identify the key characteristics that may differentiate people who did and did not respond to the intervention. Third, although many were referred by healthcare professionals and organisations supporting people with SCI, participants were treatment seeking and open to participating in a psychologically based pain management programme. It would be beneficial for future research to examine different models of providing internet-delivered interventions to adults with SCI, including the potential of direct referral into internet-delivered programmes and the optimal timing of such referral after injury. Fourth, no longitudinal data about medication use or health service use was collected in the current study, and all measurement of outcomes was self-reported. Fifth, the current study did not measure skills use, which is an important mediator of clinical outcomes, with greater adherence and skills use being associated with better clinical outcomes [37]. Finally, the intervention used in the present study has been carefully developed over several years and previously tested with $>1000$ participants $[23-25,33,34]$. Thus, the results observed in this study may not generalise to other internetdelivered pain management programmes.

In summary, the findings of the present study provide support for the potential of carefully developed internetdelivered pain management programmes for adults with SCI. High levels of engagement and satisfaction were observed and limited clinician time was required. Evidence of clinically significant improvements in levels of pain-related disability, depression, and anxiety were observed. High assessment response rates and a relatively large sample of adults with SCI was achieved, increasing confidence in the findings. Overall the findings of the current study suggest that internetdelivered interventions may represent an acceptable, efficacious, and cost-effective way of increasing access to evidence-based psychosocial care for adults with SCI.

Acknowledgements We gratefully acknowledge all of the participants for their involvement and helpful feedback throughout the research. This research was enabled by funding from the New South Wales Government's Lifetime Care and Support Authority (iCare), and the Australian National Health and Medical Research Council (NHMRC) to BFD via an Australian Public Health Fellowship. This research was also enabled via in-kind support from the New South Wales (NSW) Agency for Agency for Clinical Innovation (ACI) Pain Management and State Spinal Cord Injury Service.

Author contributions Study conception: BFD, KNP, PS, JWM, JJ, FM, and LK. Trial management: BFD. Participant recruitment: BFD, JJ, FM, and LK. Treatment protocol development: BFD and NT. Assessment and treatment: BFD. Data collection and analysis: BFD, EK, and MG. Interpretation of data: BFD, KNP, PS, JWM, JJ, LK, FM, EK, MG, and NT. Manuscript preparation: BFD, KNP, PS, JWM, JJ, LK, FM, EK, MG, and NT.

\section{Compliance with ethical standards}

Conflict of interest BFD and NT are authors and developers of the Pain Course, but derive no personal or financial benefit from it. NT and BFD are funded by the Australian Government to develop and provide a free national online assessment and treatment service, the MindSpot Clinic (www.mindspot.org.au), for people with anxiety and depression.

\section{References}

1. Benevento BT, Sipski ML. Neurogenic bladder, neurogenic bowel, and sexual dysfunction in people with spinal cord injury. Phys Ther. 2002;82:601-12. 
2. Dijkers M, Bryce T, Zanca J. Prevalence of chronic pain after traumatic spinal cord injury: a systematic review. J Rehabil Res Dev. 2009;46:13-29.

3. Jensen MP, Kuehn CM, Amtmann D, Cardenas DD. Symptom burden in persons with spinal cord injury. Arch Phys Med Rehabil. 2007;88:638.

4. Widerström-Noga EG, Felipe-Cuervo E, Broton JG, Duncan RC, Yezierski RP. Perceived difficulty in dealing with consequences of spinal cord injury. Arch Phys Med Rehab. 1999;80:580-6.

5. Siddall PJ, McClelland JM, Rutkowski SB, Cousins MJ. A longitudinal study of the prevalence and characteristics of pain in the first 5 years following spinal cord injury. Pain. 2003;103:249-57.

6. Migliorini C, Sinclair A, Brown D, Tonge B, New P. The prevalence of mood disturbance in Australian adults with chronic spinal cord injury. Intern Med J. 2015;45:1014-9.

7. Kennedy P, Rogers BA. Anxiety and depression after spinal cord injury: a longitudinal analysis. Arch Phys Med Rehab. 2000;81:932-7.

8. Cobo-Cuenca Serrano-Selva J, de la Marta-Florencio M, EstebanFuertes M, Vírseda- Chamorro M, Martín-Espinosa NM, et al. Quality of life of males with spinal cord injury and sexual dysfunction. Enferm Clínica. 2012;22:205.

9. Middleton J, Tran Y, Craig A. Relationship between quality of life and self-efficacy in persons with spinal cord injuries. Arch Phys Med Rehabil. 2007;88:1643-8.

10. Dorstyn D, Mathias J, Senson L. Efficacy of cognitive behavior therapy for the management of psychological outcomes following spinal cord injury: a meta-analysis. J Health Psychol. 2011;16:374-91.

11. Perkes SJ, Bowman J, Penkala S. Psychological therapies for the management of co-morbid depression following a spinal cord injury: a systematic review. J Health Psychol. 2014;19:1597-612.

12. Burns AS, Delparte JJ, Ballantyne EC, Boschen KA. Evaluation of an interdisciplinary program for chronic pain after spinal cord injury. Phys Med Rehabil. 2013;5:832-8.

13. Heutink M, Post MWM, Luthart P, Schuitemaker M, Slangen S, Sweers J, et al. Long-term outcomes of a multidisciplinary cognitive behavioural programme for coping with chronic neuropathic spinal cord injury pain. J Rehabil Med. 2014;46:540-5.

14. Nicholson Perry K, Nicholas MK, Middleton JW. Comparison of a pain management program with usual care in a pain management centre for people with spinal cord injury-related chronic pain. Clin J Pain. 2012;26:206-16.

15. Hogg M, Gibson S, Helou A, Degabriele J. Waiting in pain: a systematic investigation into the provision of persistent pain services in Australia. Med J Aust. 2012;196:386-90.

16. Nicholson Perry K, Nicholas MK, Middleton J. Multidisciplinary cognitive behavioural pain management programs for people with spinal cord injury: design and implementation. Dis Rehab. 2011;33:1272-80.

17. Dorstyn D, Mathias J, Linley D. Applications of tele counselling in spinal cord injury rehabilitation: a systematic review with effect sizes. Clin Rehab. 2013;27:1072-83.

18. Migliorini C, Tonge B, Sinclair A. Developing and piloting ePACT: a flexible psychological treatment for depression in people living with chronic spinal cord injury. Behav Change. 2011;28:45-54.

19. Verwer JH, van Leeuwen CMC, Bolier L, Post MWM. Feasibility of an online well-being intervention for people with a spinal cord injury: a pilot study. Spinal Cord. 2016;54:473-7.
20. Migliorini C, Sinclair A, Brown DJ, Tonge B, New P. A randomised control trial of an Internet-based cognitive behaviour treatment for mood disorder in adults with chronic spinal cord injury. Spinal Cord. 2016;54:695-701.

21. Andersson G, Titov N. Advantages and limitations of internetbased interventions for common mental disorders. World Psychiatry. 2014;13:4-11.

22. Buhrman M, Gordh T, Andersson G. Internet interventions for chronic pain including headache: a systematic review. Internet Interv. 2016;31:17-34.

23. Dear BF, Gandy M, Karin E, Staples LG, Johnston L, Fogliati VJ, et al. The Pain Course: a randomised controlled trial examining an internet-delivered pain management program when provided with different levels of clinician support. Pain. 2015;156:1920.

24. Dear BF, Gandy M, Karin E, Ricciardi T, Fogliati VJ, McDonald S, et al. The Pain Course: a randomised controlled trial comparing a remotely-delivered chronic pain management program when provided in online and workbook formats. Pain. 2017;158:1289-301.

25. Dear BF, Courtney C, Khor K, McDonald S, Ricciardi T, Gandy $\mathrm{M}$, et al. The Pain Course: exploring the feasibility of an internetdelivered pain management program when offered by a tertiary pain management service. Clin J Pain. 2018;34:505-14.

26. Pollard CA. Preliminary validity study of the pain disability index. Percept Mot Skills. 1984;59:974.

27. Kroenke K, Spitzer RL, Williams JB. The PHQ-9: validity of a brief depression severity measure. $\mathrm{J}$ Gen Intern Med. 2001;16:606-13.

28. Spitzer R, Kroenke K, Williams JB, Lowe B. A brief measure for assessing generalized anxiety disorder: the GAD-7. Arch Intern Med. 2006;166:1092-7.

29. Daut R, Cleeland CS, Flanery RC. Development of the Wisconsin Brief Pain questionnaire to assess pain in cancer and other diseases. Pain. 1983;17:197-210.

30. Nicholas MK. The pain self-efficacy questionnaire: taking pain into account. Eur J Pain. 2007;11:153-63.

31. Sullivan MJ, Bishop SR, Pivik J. The pain catastrophizing scale: development and validation. Psychol Assess. 1995;7:524.

32. Diener E, Emmons RA, Larsen RJ, Griffin S. The satisfaction with life scale. J Person Assess. 1985;49:71-5.

33. Dear BF, Titov N, Nicholson Perry K, Johnston L, Wootton BM, Terides MD, et al. The Pain Course: a randomised controlled trial of clinician-guided internet-delivered cognitive behaviour therapy program for managing chronic pain and emotional wellbeing. Pain. 2013;154:942-50.

34. Friesen LN, Hadjistavropoulos HD, Alberts NM, Schneider LH, Titov N, Dear BF. A randomised controlled trial of Internetdelivered cognitive behaviour therapy for individuals with fibromyalgia. Pain. 2017;158:593-604.

35. Hubbarb A, Ahern J, Fleisher N, Vean Der Laan M, Lippman S, Jewell N, et al. To GEE or not to GEE: comparing population average and mixed models for estimating the associations between neighbourhood risk factors and health. Epidemiology. 2010;21:467-74.

36. Little RJ, Rubin DB. Statistical analysis with missing data. Hoboken, NJ: Wiley; 2014.

37. Nicholas MK, Asghari A, Corbett M, Smeets RJEM, Wood BM, Overton $\mathrm{S}$, et al. Is adherence to pain self-management strategies associated with improved pain, depression and disability in those with disabling chronic pain?. Eur J Pain. 2012;16:93-104. 